\title{
Functional maximal strength training induces neural transfer to single-joint tasks
}

\author{
Marius S. Fimland · Jan Helgerud · Markus Gruber • \\ Gunnar Leivseth · Jan Hoff
}

\begin{abstract}
The purpose of this study was to investigate whether neural adaptations following functional multiplejoint leg press training can induce neural adaptations to the plantar flexor muscles in a single-joint contraction task. Subjects were randomised to a maximal strength training (MST) $(n=10)$ or a control group $(n=9)$. MST consisted of 24 sessions ( 8 weeks) of $4 \times 4$ repetitions of horizontal leg press using maximal intended velocity in the concentric phase with the movement ending in a plantar flexion. Neural adaptations in the soleus and gastrocnemius medialis (GM) were assessed by surface electromyographic activity and $\mathrm{V}$-waves during maximum voluntary isometric contraction (MVIC), and also by H-reflexes in the soleus during rest and 20\% MVIC. One repetition maximum leg press
\end{abstract}

M. S. Fimland $(\bigotimes)$ J. Helgerud · J. Hoff

Department of Circulation and Imaging,

Faculty of Medicine, Norwegian University of Science

and Technology, 7489 Trondheim, Norway

e-mail: marius.fimland @ntnu.no

J. Helgerud

Hokksund Medical Rehabilitation Centre, Hokksund, Norway

M. Gruber

Department of Sport Science,

University of Freiburg, Freiburg, Germany

M. Gruber

Department of Sport Science,

University of Potsdam, Potsdam, Germany

G. Leivseth · J. Hoff

Department of Physical Medicine and Rehabilitation,

St. Olav's University Hospital, Trondheim, Norway

G. Leivseth

National Center of Comprehensive Occupational Rehabilitation, Rauland, Norway increased by $44 \pm 14 \%$ (mean $\pm \mathrm{SD} ; P<0.01$ ). Plantar flexion MVIC increased by $20 \pm 14 \%(P<0.01)$, accompanied by $13 \pm 19 \%(P<0.05)$ increase in soleus, but not GM surface electromyography. Soleus $V / M_{\text {SUP }}$ increased by $53 \pm 66 \%$ and in GM by $59 \pm 64 \%(P<0.05)$. Normalised soleus $\mathrm{H}$-reflexes remained unchanged by training. No changes occurred in the control group. These results suggest that leg press MST can induce neural adaptations in a single-joint plantar flexion MVIC task.

\section{Keywords H-reflex $\cdot$ V-wave $\cdot$ Leg press $\cdot$ Strength training $\cdot$ Specificity}

\section{Introduction}

Both single-joint and multiple-joint exercises are employed in athletic strength training and are effective for improving muscular strength in the targeted muscle groups (Kraemer et al. 2002). Due to the more complex neural activation and involvement of more muscle mass (Chilibeck et al. 1998), multiple-joint strength training exercises (e.g. leg press, bench press) are generally regarded as more effective for enhancing the functional muscle strength compared to single-joint exercises (e.g. knee extension, arm curls), as most physical tasks involve multiple-joint activity (Kraemer et al. 2002; Kraemer and Ratamess 2004). It is generally recommended to incorporate both exercise types into a resistance training programme with emphasis on multiplejoint exercises (Kraemer et al. 2002). However, it can be speculated that due to the complex neural activation and vast muscle mass involvement of multiple-joint exercises, neural adaptations and thus muscular strength may be transferred to quite dissimilar single-joint tasks that could make single-joint exercises redundant in some cases. 
In line with the "specificity of training" hypothesis set forth by Sale and MacDougall (1981), a substantial degree of task specificity is generally considered to occur in response to strength training adaptations (Kraemer et al. 2002; Kraemer and Ratamess 2004). For example, some investigations have reported that an increase in dynamic lifting strength is disproportionately greater than isometric strength gains after dynamic resistance training (Rutherford and Jones 1986; Thorstensson et al. 1976). This suggests a considerable facility for learning, such as enhanced activation of the fixator muscles or improved inter-muscular coordination, which is specific for the trained task (Folland and Williams 2007). Nevertheless, several investigations employing dynamic training have demonstrated large isometric strength gains (Aagaard et al. 2002a; Adamson et al. 2008; Duclay et al. 2008). Similarly, some studies investigating the effect of various training paradigms on the central nervous system indicate that neural adaptations can be task specific (Beck et al. 2007; Schubert et al. 2008; Voigt et al. 1998), whereas other studies have not observed task specificity (Aagaard et al. 2002a, b; Duclay et al. 2008).

To investigate the plasticity of the spinal cord, the evoked Hoffmann $(\mathrm{H})$ reflex is a commonly used tool. The H-reflex has been proposed as a measure of spinal motoneuron excitability, reflecting changes at the motoneuron pool and/ or presynaptic inhibition of Ia afferent pathways (Aagaard et al. 2002b; Schieppati 1987). After heavy multi-exercise strength training, a study that used $\mathrm{H}$-reflexes to reflect training adaptations, mainly at the level of the spinal cord, demonstrated increased H-reflexes in the soleus (SOL) muscle during plantar flexion maximal voluntary isometric contraction (MVIC) (Aagaard et al. 2002b). After eccentric plantar flexion strength training, $\mathrm{H}$-reflex increases were reported in the SOL only during eccentric MVC, whereas the H-reflex was amplified during concentric, isometric and eccentric MVC in the gastrocnemius medialis (GM) muscle (Duclay et al. 2008). Aagaard et al. (2002b) and Duclay et al. (2008) did not observe any H-reflex changes in the passive state. However, enhanced $\mathrm{H}$-reflexes have been reported during low-level tonic isometric contractions [ $10 \%$ surface electromyographic (EMG) activity] in response to isometric plantar flexion strength training (Lagerquist et al. 2006). These studies have demonstrated the responsiveness of the spinal motoneuron pool to strength training.

Neural adaptations have also been shown for central drive, by measuring $\mathrm{V}$-waves during maximal contractions. The V-wave is an electrophysiological variant of the $\mathrm{H}$-reflex elicited during muscle contraction with supra-maximal stimulus intensity. It is assumed that the peak-to-peak amplitude of the $\mathrm{V}$-wave reflects the magnitude of the efferent neural drive (Aagaard et al. 2002b; Upton et al. 1971): Longitudinal studies using plantar flexion resistance training have demonstrated increased $\mathrm{V}$-wave responses in the SOL
(Aagaard et al. 2002b; Del Balso and Cafarelli 2007; Duclay et al. 2008; Gondin et al. 2006), gastrocnemius lateralis and GM (Duclay et al. 2008; Gondin et al. 2006) muscles.

If it can be assumed that multiple-joint strength training enhances muscle strength of isolated muscles, neural adaptations should be observed in single-joint tasks after multiple-joint strength training. Therefore, the aim of the present study was to investigate the transfer of neural adaptations from multiple-joint strength training to single-joint tasks. The approach was to train a functional multiple-joint leg press exercise performed with maximum intended velocity in the concentric phase with the movement ending in a plantar flexion, but to test in a non-specific single-joint plantar flexion task. It was hypothesised that heavy leg press exercise would induce changes in evoked $\mathrm{H}$-reflexes and $\mathrm{V}$-waves of the plantar flexor muscles in a single-joint task.

\section{Methods}

\section{Subjects}

A total of 19 healthy men, experienced in resistance training, but not systematically training their legs, were assigned by drawing random envelopes to either the leg press maximal strength training (MST) group $(n=10$; age $23 \pm 3$ year; height $184 \pm 6 \mathrm{~cm}$; body mass $80 \pm 7 \mathrm{~kg}$, means \pm standard deviation, SD) or a control group $(n=9$, age $24 \pm 2$ year; height $183 \pm 6 \mathrm{~cm}$; body mass $77 \pm 7 \mathrm{~kg}$ ). The study was approved by the ethics committee of the Norwegian University of Science and Technology and conformed to the standards set by the latest revision of the Declaration of Helsinki. Volunteers gave informed written consent prior to participation and had no known injuries at the time of data collection. Subjects refrained from strenuous leg activity on the day prior to each experimental session.

\section{Study overview}

Neural adaptations of the plantar flexor muscles tested in a single-joint contraction task were assessed after functional multiple-joint leg press training. The measurement protocol was completed 2 days before and 2 days after the 8-week training period. Experimental tests were conducted within 5 days before and after the training period. Measurements concerning the plantar flexors (day 2, described below) were conducted at a consistent time of day.

\section{Experimental procedure}

Prior to baseline testing, subjects attended a familiarisation session where they practiced MVICs of the plantar flexors 
(until the coefficient of variation in successive trials was $<5 \%$ ) and were familiarised with percutaneous electrical pulses delivered to the tibial nerve.

On day 1 , one repetition maximum (1RM) dynamic leg press as well as dynamic rate of force development (RFD) were measured using a horizontal leg press (Technogym, Gambettola, Italy). RFD was assessed via a force platform (9286AA, Kistler, Switzerland) attached to the foot plate of the horizontal leg press machine. After three submaximal warm-up sets, four to seven attempts were made to determine $1 \mathrm{RM}$. The heaviest load $(2.5-10 \mathrm{~kg}$ increments $)$ that could be lifted from $0^{\circ}$ to $90^{\circ}$ of knee flexion was considered as IRM. Subsequently, four to five attempts were made to determine dynamic RFD using a load equivalent to $70 \%$ of $1 \mathrm{RM}$ at pre-test. A rest of $1 \mathrm{~min}$ was allowed between attempts. Only the best attempts (IRM: highest load lifted, $\mathrm{kg}$; dynamic RFD: highest RFD $\left(\mathrm{N} \mathrm{s}^{-1}\right)$ from 10 to $90 \%$ of peak force) were considered for analysis. Standardised verbal encouragement was provided by the same investigator at all trials.

On day 2 (see Fig. 1), all measurements concerning the plantar flexors were conducted: MVICs, H-reflex excitability at rest and during submaximal activation, and $\mathrm{V}$-waves. The plantar flexors only performed isometric contractions. Five MVICs were performed to determine the voluntary plantar flexion strength. The H-reflex stimulus intensity was controlled by a small $M$-wave, $10 \pm 2.5 \%$ of the maximal $\mathrm{M}$-wave $\left(\mathrm{M}_{\mathrm{MAX}}\right)$. The tonic contraction intensity used for H-reflexes evoked during submaximal activation was $20 \%$ of MVIC on the same day. Finally, 8-10 V-waves were manually elicited during MVICs $\sim 2 \mathrm{~s}$ after the onset of contraction.

Subjects were seated slightly reclined in a chair mounted on a solid wooden platform with their right foot placed in a custom-made isometric ankle dynamometer made of steel

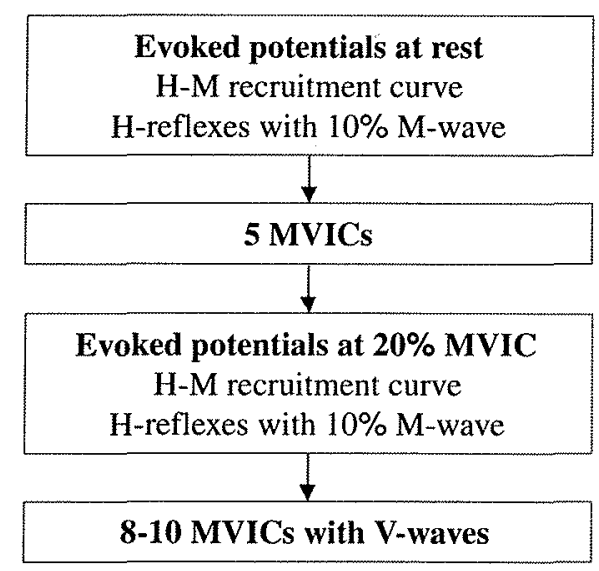

Fig. 1 Day 2 of the experimental protocol. MVIC maximum voluntary isometric contraction. Small $M$-wave was $10 \pm 2.5 \%$ of the highest $\mathrm{M}$-wave evoked in the same condition and plexiglass. The axis of rotation of the foot plate was aligned with the anatomical axis of the ankle. Rigid straps secured the heel and forefoot to the foot plate with the ankle at $90^{\circ}$. The thigh, hip and back were secured with broad velcro straps and held the subject in a constant position with the knee flexed at $80^{\circ}$ from full extension and the hip at $90^{\circ}$. Visual feedback of submaximal and maximal force was continuously provided on a screen in front of the subject. MVICs were performed at a rate of one per minute and each attempt lasted until the subject had received the supramaximal stimulus. Standardised verbal encouragement was provided by the same investigator at all trials.

\section{Electromyography and force recordings}

After preparation of the skin (day 2), pairs of bipolar Ag/ $\mathrm{AgCl}$ surface electrodes (Ambu M-00-S, Ballerup, Denmark) were placed on the SOL, GM, tibialis anterior (TA) and vastus lateralis (interelectrode distance $25 \mathrm{~mm}$ ) muscles of the right leg. Anatomical landmarks were used to ensure the same placement during pre- and post-tests. Subjects performed preliminary contractions, and the shape of the $\mathrm{M}$-waves and $\mathrm{H}$-reflexes were monitored to ensure optimal placement of the stimulating and recording electrodes. EMG was sampled (ME6000 Biomonitor, Mega Electronics LTD, Kuopio, Finland) at $2 \mathrm{kHz}$, CMRR: $110 \mathrm{~dB}$, amplified and band-pass filtered $(8-500 \mathrm{~Hz})$ prior to being stored on a personal computer. To calculate voluntary activation levels, the amplified raw EMG signals were converted to the root mean square (RMS) values of a $500 \mathrm{~ms}$ epoch coinciding with peak force. The three highest MVICs (EMG amplitude and peak force) were analysed and averaged.

The isometric forces exerted by the plantar flexors was recorded by a force-transducer (Model 363-D3-50-20P1, Revere Transducers, Tustin, CA, USA) that responds linearly within a load range of $0-250 \mathrm{~kg}$ with a reproducibility error of $0.1 \%$ attached to the ankle dynamometer. Plantar flexion force and dynamic leg press RFD were recorded at $1 \mathrm{kHz}$ and digitally low-pass filtered $(10 \mathrm{~Hz})$. Plantar flexion force and EMG were analysed using MegaWin software (Mega Electronics LTD, Kuopio, Finland). Dynamic RFD $\left(\mathrm{N} \mathrm{s}^{-1}\right)$ was calculated from the $10-90 \%$ of peak force in the concentric phase of the lift (Hoff et al. 2007) and analysed using Bioware v3.06b software (Kistler, Switzerland). The plantar flexion force was multiplied with the consistent moment arm length of $0.15 \mathrm{~m}$ (measured from the heel block to the centre of the force transducer) to attain torque.

Stimulation and analysis of $\mathrm{H}$-reflexes, $\mathrm{V}$-waves and contractile properties

$\mathrm{H}$-reflexes and $\mathrm{V}$-waves were evoked in the tibial nerve using a constant-current stimulator (DS7, Digitimer, 
Welwyn Garden City, UK). A 1-ms square wave stimulus was applied to the posterior tibial nerve in the popliteal fossa with gel-coated bipolar felt pad electrodes ( $8 \mathrm{~mm}$ diameter, $25 \mathrm{~mm}$ between tips; Digitimer, Welvyn Garden City, UK). The cathode was placed medial to the anode to avoid anodal block (Pierrot-Deseilligny and Burke 2005). After the optimal site of stimulation had been determined, rigid taping and straps secured a constant pressure and position throughout the experimental session. For the stimulation of $\mathrm{V}$-waves, at least $150 \%$ of the current intensity needed to evoke $M_{\text {MAX }}$ was employed.

H-reflex excitability was determined by averaging at least five $\mathrm{H}$-reflexes with a corresponding small $\mathrm{M}$-wave $\left(10 \pm 2.5 \%\right.$ of $\left.\mathrm{M}_{\mathrm{MAX}}\right)$ both at rest and during muscle activation (20\% MVIC). These values were normalised by the $\mathrm{M}_{\mathrm{MAX}}$ evoked in the same condition. To determine the level of efferent neural drive, V-waves were obtained in the SOL and GM during MVIC and normalised by the corresponding superimposed $M$-wave $\left(M_{S U P}\right)$. To ensure that measurement conditions were stable, only $V$-waves with a corresponding $\mathrm{M}_{\text {SUP }}$ of $>90 \%$ of the highest $\mathrm{M}_{\text {SUP }}$ were included.

To assess the contractile properties of the plantar flexors, the following twitch characteristics were determined from the mechanical twitch associated with $\mathrm{M}_{\mathrm{MAX}}$ : (1) peak twitch, defined as the difference from baseline to peak twitch torque, (2) time to peak twitch torque, defined as the time between onset ( $3 \%$ of peak twitch) and peak twitch torque, and (3) half-relaxation time, defined as the time from peak twitch torque to half-twitch torque. Three twitches were analysed and averaged for each subject.

\section{Training intervention}

The training group performed an 8-week training regime (three non-consecutive days/week, i.e. 24 sessions) supervised in our laboratory. The strength training sessions consisted of four sets of four repetitions, separated by $3 \mathrm{~min}$ of rest between sets. The emphasis was put on lowering the weight in a controlled manner until the knee joints were at $90^{\circ}$, short stop ( $\left.\sim 0.5 \mathrm{~s}\right)$ and then maximal mobilisation of force in the concentric movement with the movement ending in a plantar flexion. This is a common type of training exercise in our laboratory (Hoff et al. 2007; Storen et al. 2008 ). The training load corresponded to $85-90 \%$ of $1 \mathrm{RM}$, and to stay within this range it was increased by $5-10 \mathrm{~kg}$ when the target repetitions could be completed for all sets. Both the MST and control group were encouraged to continue their normal daily activities, but asked not to commence a new training programme between the pre- and post-tests.

\section{Statistical analysis}

As the assumption of normality could not be confirmed for all variables pre- to post-test changes were assessed by the Wilcoxon signed-rank test for paired samples and differences between groups were assessed by the Mann-Whitney $U$ test. Data are expressed as means $\pm \mathrm{SD}$ in the text and tables, and as means $\pm \mathrm{SE}$ in figures. A $P$ value of $\leq 0.05$ was considered to be statistically significant.

\section{Results}

Baseline testing and exercise training adherence

No differences could be observed between the two groups in any of the pre-test measures $(P=0.149-1$; Figs. 2, 3;

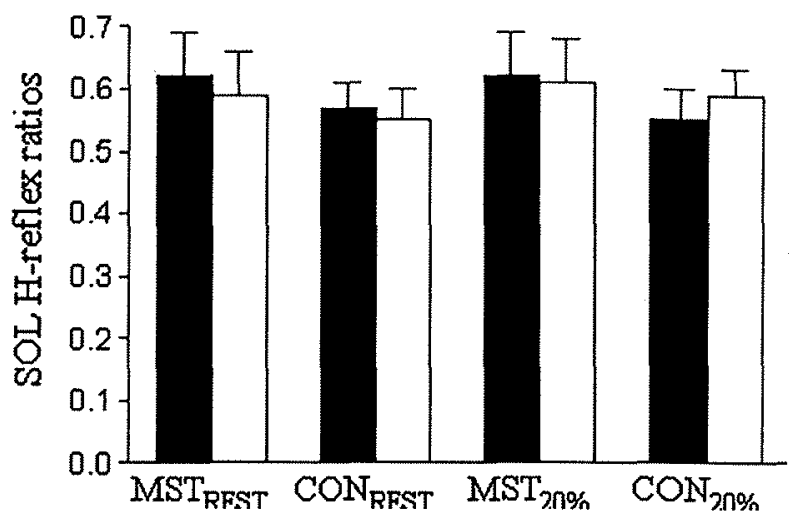

Fig. 2 Soleus (SOL) $H / M_{M A X}$ ratios obtained with a corresponding small M-wave $\left(10 \pm 2.5 \% \mathrm{M}_{\mathrm{MAX}}\right)$ at rest and during submaximal activation (20\% MVIC; denoted by $20 \%$ ) in the maximal strength trained $(M S T)$ and control group (CON), before (black bars) and after (white bars) the 8-week period

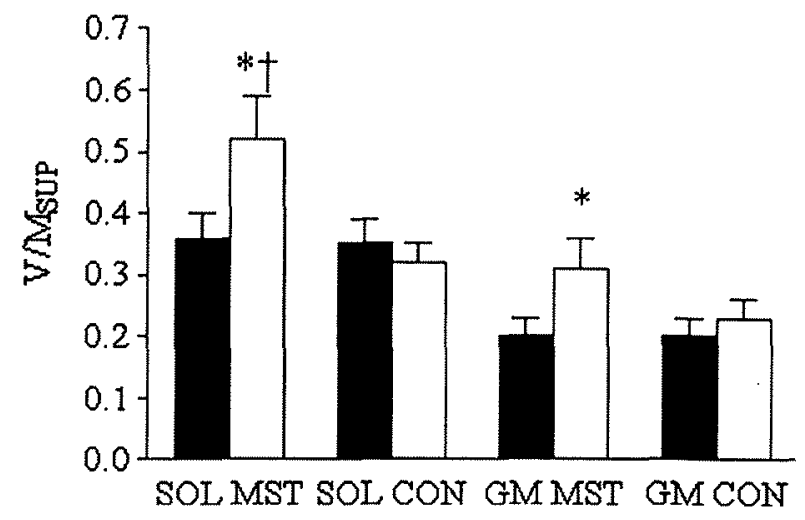

Fig. 3 Soleus (SOL) and medial gastrocnemius $(G M) \mathrm{V} / \mathrm{M}_{\mathrm{SUP}}$ ratios obtained during maximum voluntary isometric contractions $(M V I C)$ in the maximal strength trained $(M S T)$ and control group (CON), before (black bars) and after (white bars) the 8 -week period. $* P<0.05$, different from pre-test; $P<0.05$, different between groups. Means $\pm \mathrm{SE}$ 
Table 1 Strength, contractile properties and body mass before and after the 8-week period of maximal strength training or normal activity

\begin{tabular}{|c|c|c|c|c|}
\hline & \multicolumn{2}{|c|}{ Maximal strength training group } & \multicolumn{2}{|c|}{ Control group } \\
\hline & Pre & Post & Pre & Post \\
\hline Leg press 1RM (kg) & $165(26)$ & $236(29)^{\# \dagger}$ & $180(22)$ & $177(19)$ \\
\hline Leg press RFD $\left(\mathrm{N} \mathrm{s}^{-1}\right)$ & $2,015(684)$ & $4,596(1,661)^{\# !}$ & $2,138(559)$ & $2,187(618)$ \\
\hline MVIC ( $\mathrm{N} \mathrm{m})$ & $133(18)$ & $160(20)^{\#}$ & $151(30)$ & $149(32)$ \\
\hline Peak twitch $(\mathrm{N} \mathrm{m})$ & $25(3)$ & $27(3)$ & $28(5)$ & $27(6)$ \\
\hline Time to peak twitch (ms) & $112(10)$ & $109(11)$ & $107(14)$ & $108(13)$ \\
\hline Twitch half-relaxation time (ms) & $114(11)$ & $116(9)$ & $111(12)$ & $113(9)$ \\
\hline Body mass $(\mathrm{kg})$ & $79.7(7.1)$ & $80.3(8.7)$ & $77.0(7.0)$ & $77.4(6.8)$ \\
\hline
\end{tabular}

Data are means (SD)

IRM 1 repetition maximum, $R F D$ dynamic rate of force development, $M V I C$ plantar flexion maximum voluntary isometric contraction

${ }^{\#} P<0.01$ from pre- to post-test; ${ }^{\dagger} P<0.001$ between groups

Table $2 \mathrm{EMG}_{\mathrm{RMS}}$ activity during plantar flexion MVIC and absolute evoked peak-to-peak amplitude potentials (mV) of the soleus (SOL) and medial gastrocnemius (GM) muscles

\begin{tabular}{|c|c|c|c|c|c|c|c|c|}
\hline & \multicolumn{4}{|c|}{ Maximal strength training group } & \multicolumn{4}{|c|}{ Control group } \\
\hline & \multicolumn{2}{|l|}{$\mathrm{SOL}$} & \multicolumn{2}{|l|}{ GM } & \multicolumn{2}{|l|}{ SOL } & \multicolumn{2}{|l|}{ GM } \\
\hline & Pre & Post & Pre & Post & Pre & Post & Pre & Post \\
\hline $\mathrm{EMG}_{\mathrm{RMS}}$ & $0.26(0.06)$ & $0.29(0.08)^{*}$ & $0.18(0.06)$ & $0.21(0.05)$ & $0.28(0.08)$ & $0.26(0.05)$ & $0.21(0.05)$ & $0.19(0.04)$ \\
\hline $\mathrm{H}_{\mathrm{REST}}$ & $4.79(2.59)$ & $4.99(2.84)$ & NA & NA & $4.53(1.20)$ & $4.67(1.97)$ & NA & NA \\
\hline $\mathrm{M}_{\mathrm{MAX}}$ & $7.56(2.81)$ & $7.65(2.68)$ & NA & NA & $8.01(1.45)$ & $8.27(1.97)$ & NA & NA \\
\hline $\mathrm{H}_{20 \%}$ & $5.67(2.82)$ & $5.76(2.75)$ & NA & NA & $4.83(1.55)$ & $5.57(1.63)$ & NA & NA \\
\hline $\mathrm{M}_{\mathrm{MAX} 20 \%}$ & $8.83(2.47)$ & $8.97(1.86)$ & NA & NA & $8.94(1.55)$ & $9.34(1.46)$ & NA & NA \\
\hline V-wave & $3.39(1.24)$ & $4.91(2.17)^{*}$ & $1.65(0.48)$ & $2.53(1.10)^{* \dagger}$ & $3.46(1.54)$ & $3.19(1.07)$ & $1.58(0.84)$ & $1.37(0.51)$ \\
\hline $\mathrm{M}_{\text {SUP }}$ & $9.47(1.76)$ & $9.46(1.44)$ & $8.65(1.74)$ & $8.60(1.38)$ & $9.72(1.35)$ & $9.78(1.67)$ & $8.00(1.45)$ & $7.47(1.21)$ \\
\hline
\end{tabular}

Data are means (SD)

NA not considered for analysis, MVIC plantar flexion maximum voluntary isometric contraction, $20 \%$ measurements obtained during a tonic $20 \%$ plantar flexion MVIC, $H$ H-reflexes

$* P<0.05$, from pre- to post-test; ${ }^{\dagger} P<0.05$, different between groups

Tables 1,2). The subjects completed $100 \%$ of the assigned training.

\section{Strength parameters and EMG activity}

The control group revealed no significant changes in strength- or EMG activity-related variables (Tables. 1, 2), whereas the leg press MST group had significant increase in all measured strength parameters (Table 1). Dynamic leg press $1 \mathrm{RM}$ increased by $44 \pm 14 \%(P=0.005)$, whereas dynamic leg press RFD increased by $157 \pm 135 \%$ $(P=0.009)$. Plantar flexion MVIC increased by $20 \pm 14 \%$ $(P=0.005)$ after leg press MST. The increase in MVIC for the leg press MST group was accompanied by a $13 \pm 19 \%$ $(P=0.047)$ increase for SOL, but not for $\mathrm{GM} E \mathrm{EMG}_{\mathrm{RMS}}$ $(P=0.114)$ (Table 2). During plantar flexion MVIC, no changes could be observed in absolute $(\mathrm{mV})$ antagonist TA
$\mathrm{EMG}_{\mathrm{RMS}}$ activity for the MST (pre: $0.042 \pm 0.012$, post: $0.036 \pm 0.010 ; P=0.203$ ) or the control group (pre: $0.042 \pm 0.014$, post: $0.035 \pm 0.008 ; P=0.183$ ).

\section{Evoked potentials}

No change could be observed for the control group in any of the evoked potentials or calculated reflex ratios between pre- and post-tests (Table 2, Figs. 2, 3). In the leg press MST group, the SOL H-reflex recordings normalised by $\mathrm{M}_{\mathrm{MAX}}$ did not change at rest $(P=0.575)$ or during submaximal (20\% MVIC) activation $(P=0.407)$ (Table 2, Fig. 3). During MVIC, the V/M $\mathrm{M}_{\text {SuP }}$ ratios increased by $53 \pm 66$ and $59 \pm 64 \%$ in the SOL $(P=0.047)$ and GM $(P=0.021)$, respectively (Table 2 ; Fig. 3 ). The level of voluntary force at the onset of the V-wave stimuli, relative to MVIC on the same day, was similar between sessions in both the control 
group (pre: $96.5 \pm 1.7 \%$, post: $97.0 \pm 1.7 \% ; P=0.441$ ) and the leg press MST group (pre: $96.3 \pm 2.1 \%$, post: $97.4 \pm 1.6 \% ; P=0.241$ ). Examples of SOL and GM $\mathrm{V}$-waves and associated $\mathrm{M}_{\text {Sup }}$ before and after the 8-week training period are demonstrated in Fig. 4 for one subject.

\section{Contractile properties}

The peak twitch, time to peak twitch and half-relaxation time remained unchanged from pre- to post-tests in the leg press MST $(P=0.220-0.285)$ and in the control group $(P=0.374-0.550$; Table 1).

\section{Discussion}

This study demonstrated that 8 weeks of heavy leg press exercise with a focus on maximal intended velocity in the concentric phase (MST) increased isolated isometric plantar flexion strength by $20 \%, \sim$ half of the enhancement in the multiple-joint training exercise (44\%), and was accompanied by increased $\mathrm{EMG}_{\mathrm{RMS}}$ activity of the SOL, but not GM muscle during plantar flexion MVIC. After leg press MST, the V/M $\mathrm{M}_{\text {SuP }}$ ratios increased in both SOL and GM, whereas no change occurred in normalised SOL H-reflexes at rest or during submaximal activation. Thereby, the

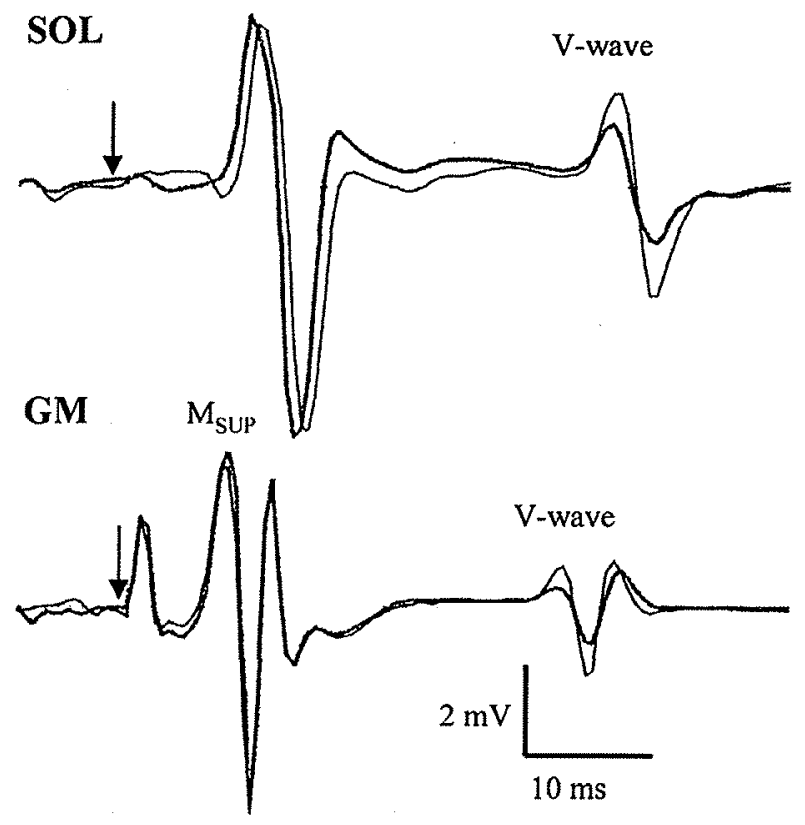

Fig. 4 Soleus (SOL) and medial gastrocnemius (GM) V-wave and superimposed $M$-wave $\left(M_{\text {SuP }}\right.$ ) recordings (means of at least four trials) from one subject in the maximal strength training group obtained before (thick line) and after (thin line) the 8-week training period. Arrows indicate time of stimulation. Note the consistent $\mathrm{M}_{\text {Sup }}$ and increased $\mathrm{V}$-wave amplitudes after strength training present study demonstrated that functional multiple-joint leg press training enhances neural drive in a single-joint plantar flexion MVIC task.

\section{Methodological considerations}

In the present study, great care was taken for the extraneous factors known to influence H-reflexes, such as posture, voluntary movement, noise, light and previous activity (Zehr 2002). To avoid the influence of $\mathrm{H}$-reflex potentiation (Folland et al. 2008), resting H-reflexes were obtained prior to MVICs. To ensure constant stimulus intensity, H-reflexes were evoked with a corresponding small $\mathrm{M}$-wave $(\sim 10 \%$ of $\mathrm{M}_{\mathrm{MAX}}$ ) in the SOL muscle. As this stimulus intensity produced variable $M$-wave amplitudes in the GM muscle amongst subjects, GM H-reflexes were not considered in the analysis of H-reflex excitability. The H-reflex stimulus intensity was optimised for the SOL, as this muscle is generally preferred for H-reflex measurements (Pierrot-Deseilligny and Burke 2005; Schieppati 1987). Many previous exercise experiments have used the $\mathrm{H}_{\text {MAX }} / \mathrm{M}_{\text {MAX }}$ ratio as an index of $\mathrm{H}$-reflex excitability, however, $\mathrm{H}$-reflexes are more sensitive to facilitation or inhibition on the ascending limb of the recruitment curve (for review see Pierrot-Deseilligny and Mazevet (2000)). With the stimulus intensity employed in the current study, SOL H-reflexes were collected on the ascending part of the H-reflex curve in eight out of ten subjects in the leg press MST group. Excluding the two subjects where H-reflexes were obtained on the descending part of the recruitment curve did not change the outcome of the analysis.

Effects of leg press MST on single-joint plantar flexion strength, EMG activity and contractile properties

We are not aware of studies that have reported increased isolated strength of the plantar flexor muscles after multiple-joint leg press training. Some previous reports suggest that improvements in strength after resistance training are largely limited to specific movement patterns (Jones and Rutherford 1987; Thorstensson et al. 1976), although other studies have reported significant gains after non-specific training (Aagaard et al. 2002b; Andersen et al. 2005). However, the studies by Aagaard and Andersen included several exercises and a high volume of work completed. Therefore, a $20 \%$ increase in isometric plantar flexion strength after leg press training in the present study is quite remarkable and is similar to that reported by others after specific isometric plantar flexion strength training (Del Balso and Cafarelli 2007; Holtermann et al. 2007). Heavy weights combined with the maximal mental effort in the present study, which substantially activated the plantar flexors during the leg press exercise, likely explains the relatively 
large improvements, despite the quite non-specific training and testing tasks in the present study.

The increase in strength was accompanied by a significant $13 \%$ increase in $\mathrm{EMG}_{\mathrm{RMS}}$ activity in the SOL muscle. As the EMG signal is a complex outcome of motor unit recruitment and firing frequency (Aagaard 2003; Farina et al. 2004), one or both of these mechanisms could have contributed to the increase in $\mathrm{EMG}_{\mathrm{RMS}}$ activity after leg press MST. Still, it should be noted that changes in EMG interference activity could be influenced by altered patterns of motor unit action potential summation (Farina et al. 2004), due to changes in muscle fibre morphology and/or muscle architecture. As there was no change in the contractile properties of the plantar flexors, we assume that increases in strength and EMG are mainly related to neural adaptations, although we cannot completely exclude the possibility of morphological influences as more sensitive measurements of muscle morphology (i.e. muscle biopsies, magnetic resonance imaging or computerised tomography) were not conducted.

\section{No effects of leg press MST on H-reflexes}

During rest and tonic contractions of $20 \% \mathrm{MVIC}$, we found no modulation of H-reflex amplitudes after leg press MST. The finding that $\mathrm{H}$-reflexes obtained in a relaxed muscle remained unchanged after resistance training is consistent with several other investigations (Aagaard et al. 2002b; Del Balso and Cafarelli 2007; Duclay et al. 2008; Scaglioni et al. 2002) and suggests that at rest the motoneuron pool is not affected by training.

During muscle activation, the results are discrepant. After multi-exercise strength training, Aagaard et al. (2002b) reported a $\sim 20 \%$ increase in the $\mathrm{H}$-reflex at a stimulus intensity corresponding to $20 \%$ of $\mathrm{M}_{\mathrm{MAX}}$ whereas Gondin et al. (2006) observed no change in the $H_{\text {SUP }} / M_{\text {SuP }}$ ratio after neuromuscular electrical stimulation training. H-reflexes were evoked during MVIC in both studies.

Furthermore, obtained during tonic submaximal activation (10\% of maximal EMG activity), Lagerquist et al. (2006) reported no change in the $H_{M A X} / M_{M A X}$ ratio whereas a significant increase in $\mathrm{H}$-reflex amplitude could be observed on the ascending part of the recruitment curve $\left(\mathrm{M}\right.$-wave at $5 \%$ of $\left.\mathrm{M}_{\mathrm{MAX}}\right)$ in response to isometric strength training. In contrast to the findings by Lagerquist et al., Holtermann et al. (2007) reported increased H-reflexes during 20 and $60 \% \mathrm{MVIC}$, with a stimulation intensity that produced M-waves at $20 \%$ of $\mathrm{M}_{\mathrm{MAX}}$, which would likely be on the descending part of the H-reflex recruitment curve for several subjects. Del Balso and Cafarelli (2007) did not observe a change in $\mathrm{H}$-reflex excitability as determined by dividing the recruitment curve of the H-reflex slope by the M-wave slope during a tonic $10 \%$ MVIC. In the present study, H-reflexes obtained during $20 \%$ MVIC with a stimulus intensity that evoked concomitant $\mathrm{M}$-waves $\sim 10 \%$ of $M_{\text {MAX }}$, did not change in response to functional leg press training. These inconsistencies may be explained by the different training modalities or methodological differences, such as the different levels of muscular contractions employed or the different stimulus intensities used to evoke H-reflexes. For example, it is possible that the recruitment and/or firing frequency of the slow-twitch motoneurons, which are primarily activated by the H-reflex volley of action potentials (Schieppati 1987), were not affected by the very heavy strength training performed in the present study. Thus, unchanged $H$-reflex excitability in this study suggests that the SOL H-reflex pathway is not influenced by heavy multiple-joint leg press training, but this does not preclude adaptations of other spinal pathways. More research seems warranted to elucidate the confusing differences reported in literature.

\section{Effects of leg press MST on V-wave responses}

For the first time, increased SOL and GM V/M responses have been observed after a training intervention where the plantar flexor muscles did not undertake isolated training. This finding demonstrates that a multiple-joint exercise can induce adaptations of neural control of single muscles in a quite non-specific task. Other longitudinal investigations have reported similar enhancements in $\mathrm{V}$-wave amplitudes after different training protocols. However, they all employed isolated training of the plantar flexors. For example, Aagaard et al. (2002b) reported a 55\% increase in normalised SOL V-wave responses after a 14-week heavy, multi-exercise training programme, including dynamic seated calf raises. Similarly, Del Balso and Cafarelli (2007) reported that 4 weeks of isometric plantar flexion exercise resulted in increased normalised SOL V-wave responses $(+57 \%)$. Increased $\mathrm{V} / \mathrm{M}_{\mathrm{SuP}}$ responses were also recently demonstrated in the $S O L$, gastronemius lateralis and GM muscles $(+81,+76$, and $+97 \%$, respectively) after 5 weeks of neuromuscular isometric electrical stimulation exercise of the ankle plantar flexors (Gondin et al. 2006).

Increased $\mathrm{V} / \mathrm{M}_{\text {Sup }}$ responses after leg press MST suggest increased efferent neural drive from spinal motoneurons to the muscles. This enhanced efferent neural drive can be measured due to the proportional removal of antidromic impulses, allowing a larger part of the evoked reflex volley of action potentials to pass to the muscle, resulting in a larger V-wave. The increase in efferent neural drive is likely caused by enhanced corticospinal drive, motoneuron excitability and/or changes in presynaptic inhibition mediating increased motoneuron recruitment and/or firing frequency (Aagaard et al. 2002b). Rearranging the mathematical equation formulated by Upton et al. (1971), Aagaard 
et al. (2002b) suggested that the increase in V-wave amplitude after heavy resistance training was likely caused by an increase in motoneuron firing frequency mediated by enhanced motor drive from higher centres. There were no changes at rest or during submaximal activation, and thus it can be assumed that adaptations are restricted to maximal contractions. Hence, it could be that the maximal mental effort involved in multiple-joint strength training induces adaptations presumably at the supraspinal level, which can be transferred to other maximal motor tasks involving some of the same muscles.

In conclusion, augmented $\mathrm{V} / \mathrm{M}_{\mathrm{Sup}}$ responses and SOL EMG activity during plantar flexion MVIC suggest that neural mechanisms contributed to the strength improvement of the plantar flexor muscles in response to 8 weeks of leg press MST. These findings demonstrate that functional multiple-joint strength training, performed with maximal intended velocity in the concentric phase, can induce neural adaptations to quite non-specific maximal muscle tasks.

Acknowledgments The authors thank Lina Bull, Henrik Loe and Athanasios Triantafyllou for valuable help during the training and testing, and Ketil Jensen and Ole Støren for technical assistance. We are indebted to the subjects who took part in the study. We are grateful to the reviewers for improving the manuscript

\section{References}

Aagaard $P$ (2003) Training-induced changes in neural function. Exerc Sport Sci Rev 31:61-67. doi:10.1097/00003677-20030400000002

Aagaard P, Simonsen EB, Andersen JL, Magnusson P, Dyhre-Poulsen $P(2002 a)$ Increased rate of force development and neural drive of human skeletal muscle following resistance training. J Appl Physiol 93:1318-1326

Aagaard P, Simonsen EB, Andersen JL, Magnusson P, Dyhre-Poulsen P (2002b) Neural adaptation to resistance training: changes in evoked $V$-wave and H-reflex responses. J Appl Physiol 92:2309-2318

Adamson M, Macquaide N, Helgerud J, Hoff J, Kemi OJ (2008) Unilateral arm strength training improves contralateral peak force and rate of force development. Eur J Appl Physiol 103:553-559. doi: 10.1007/s00421-008-0750-6

Andersen LL, Andersen JL, Magnusson SP, Suetta C, Madsen JL, Christensen LR, Aagaard P (2005) Changes in the human muscle force-velocity relationship in response to resistance training and subsequent detraining. J Appl Physiol 99:87-94. doi:10.1152/ japplphysiol.00091.2005

Beck S, Taube W, Gruber M, Amtage F, Gollhofer A, Schubert M (2007) Task-specific changes in motor-evoked potentials of lower limb muscles after different training interventions. Brain Res 1179:51-60, doi: 10.1016/j.brainres.2007.08.048

Chilibeck PD, Calder AW, Sale DG, Webber CE (1998) A comparison of strength and muscle mass increases during resistance training in young women. Eur $\mathbf{J}$ Appl Physiol Occup Physiol 77:170-175. doi: $10.1007 / \mathrm{s} 004210050316$

Del Balso C, Cafarelli E (2007) Adaptations in the activation of human skeletal muscle induced by short-term isometric resistance training. J Appl Physiol 103:402-411. doi:10.1152/japplphysiol. 00477.2006
Duclay J, Martin A, Robbe A, Pousson M (2008) Spinal reflex plasticity during maximal dynamic contractions after eccentric training. Med Sci Sports Exerc 40:722-734. doi:10.1249/MSS.0b013e318 $16184 \mathrm{dc}$

Farina D, Merletti R, Enoka RM (2004) The extraction of neural strategies from the surface EMG. J Appl Physiol 96:1486-1495. doi: 10.1152/japplphysiol.01070.2003

Folland JP, Williams AG (2007) The adaptations to strength training: morphological and neurological contributions to increased strength. Sports Med 37:145-168. doi:10.2165/00007256-2007 37020-00004

Folland JP, Wakamatsu T, Fimland MS (2008) The influence of maximal isometric activity on twitch and $\mathrm{H}$-reflex potentiation, and quadriceps femoris performance. Eur J Appl Physiol 104:739 748. doi:10.1007/s00421-008-0823-6

Gondin J, Duclay J, Martin A (2006) Soleus- and gastrocnemii-evoked $V$-wave responses increase after neuromuscular electrical stimulation training. J Neurophysiol 95:3328-3335. doi:10.1152/ jn.01002.2005

Hoff J, Tjonna AE, Steinshamn S, Hoydal M, Richardson RS, Helgerud $\mathrm{J}$ (2007) Maximal strength training of the legs in COPD: a therapy for mechanical inefficiency. Med Sci Sports Exerc 39:220-226. doi: $10.1249 / 01 . \mathrm{mss} .0000246989 .48729 .39$

Holtermann A, Roeleveld K, Engstrom M, Sand T (2007) Enhanced $\mathrm{H}$-reflex with resistance training is related to increased rate of force development. Eur J Appl Physiol 101:301-312. doi:10.1007/ s00421-007-0503-y

Jones DA, Rutherford OM (1987) Human muscle strength training: the effects of three different regimens and the nature of the resultant changes. J Physiol 391:1-11

Kraemer WJ, Ratamess NA (2004) Fundamentals of resistance training: progression and exercise prescription. Med Sci Sports Exerc 36:674-688

Kraemer WJ, Adams K, Cafarelli E, Dudley GA, Dooly C, Feigen baum MS, Fleck SJ, Franklin B, Fry AC, Hoffman JR, Newton RU, Potteiger J, Stone MH, Ratamess NA, Triplett-McBride T (2002) American College of Sports Medicine position stand. Progression models in resistance training for healthy adults. Med Sci Sports Exerc 34:364-380

Lagerquist O, Zehr EP, Docherty D (2006) Increased spinal reflex excitability is not associated with neural plasticity underlying the cross-education effect. J Appl Physiol 100:83-90

Pierrot-Deseilligny E, Burke D (2005) The circuitry of the human spinal cord: its role in motor control and movement disorders. Cambridge University Press, New York

Pierrot-Deseilligny E, Mazevet D (2000) The monosynaptic reflex: a tool to investigate motor control in humans. Interest and limits. Clin Neurophysiol 30:67-80

Rutherford OM, Jones DA (1986) The role of learning and coordination in strength training. Eur J Appl Physiol Occup Physiol 55:100-105

Sale D, MacDougall D (1981) Specificity in strength training: a review for the coach and athlete. Can J Appl Sport Sci 6:87-92

Scaglioni G, Ferri A, Minetti AE, Martin A, Van Hoecke J, Capodaglio P, Sartorio A, Narici MV (2002) Plantar flexor activation capacity and $H$ reflex in older adults: adaptations to strength training J Appl Physiol 92:2292-2302

Schieppati M (1987) The Hoffmann reflex: a means of assessing spinal reflex excitability and its descending control in man. Prog Neuro biol 28:345-376

Schubert M, Beck S, Taube W, Amtage F, Faist M, Gruber M (2008) Balance training and ballistic strength training are associated with task-specific corticospinal adaptations. Eur J NeuroSci 27:2007-2018

Storen O, Helgerud J, Stoa EM, Hoff J (2008) Maximal strength training improves running economy in distance runners. Med Sci Sports Exerc 40:1087-1092 
Thorstensson A, Hulten B, von Dobeln W, Karlsson J (1976) Effect of strength training on enzyme activities and fibre characteristics in human skeletal muscle. Acta Physiol Scand 96:392-398

Upton AR, McComas AJ, Sica RE (1971) Potentiation of "late" responses evoked in muscles during effort. $J$ Neurol Neurosurg Psychiatry 34:699-711
Voigt M, Chelli F, Frigo C (1998) Changes in the excitability of soleus muscle short latency stretch reflexes during human hopping after 4 weeks of hopping training. Eur J Appl Physiol Occup Physiol $78: 522-532$

Zehr PE (2002) Considerations for use of the Hoffmann reflex in exercise studies. Eur J Appl Physiol 86:455-468 